PÉREZ VIDAL Alejandro, Bartolomé J. Gallardo. Satira, pensamiento y politica, Badajoz 1999, Editora Regional de Extremadura, 400 p.

Lluís Roura

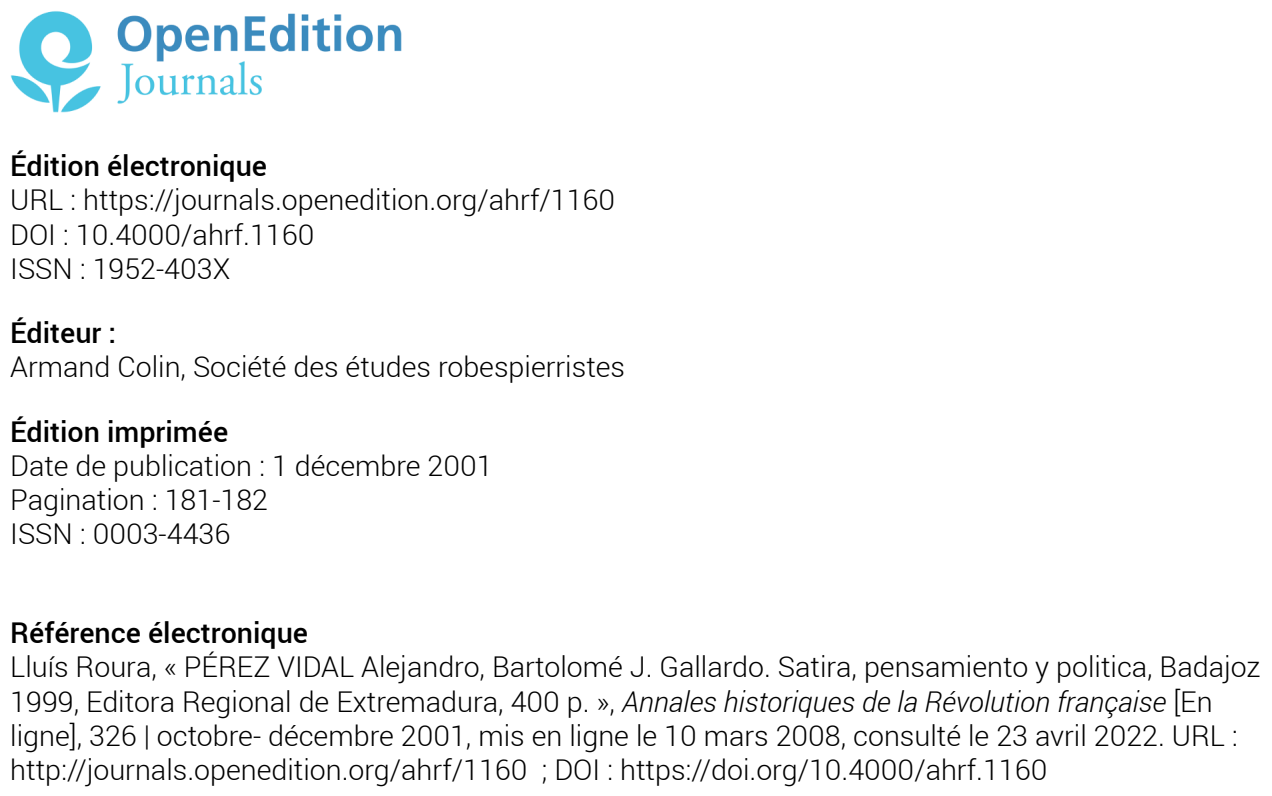

Ce document a été généré automatiquement le 23 avril 2022.

Tous droits réservés 


\title{
PÉREZ VIDAL Alejandro, Bartolomé J. Gallardo. Satira, pensamiento y politica, Badajoz 1999, Editora Regional de Extremadura, $400 \mathrm{p}$.
}

\author{
Lluís Roura
}

1 Quelques années après l'édition critique d'une des œuvres les plus significatives de la polémique idéologique autour de la révolution libérale espagnole des Cortes de Cadix (1), Alejandro Pérez - sans doute le meilleur connaisseur de Bartolomé Gallardo nous offre cette étude approfondie du publiciste et de son œuvre.

Bien qu'A. Pérez nous avertisse qu'il ne s'agit pas de la monographie générale que requiert la trajectoire intellectuelle et littéraire de Gallardo, il faut reconnaître que son travail n'en reste pas éloigné. Il s'agit fondamentalement d'une étude sur la portée et la signification des écrits satiriques de Gallardo, ainsi que de son rapport avec les contextes littéraires, idéologiques, historiques et biographiques dans lesquels ils ont paru. À travers Gallardo, donc, l'auteur nous offre une approche singulière de l'histoire du premier libéralisme espagnol.

De façon très graphique, A. Pérez souligne l'intérêt de l'œuvre satirique de Gallardo, et de la satire en général, dans la révolution espagnole: «Ainsi que dans les gravures de Goya on y trouve la crise de la culture des Lumières et de la vie espagnole avec un degré d'éloquence pareil à celui de ses œuvres de genre classique [...] dans la nouvelle époque littéraire du romantisme - dès le Gallardo de la guerre antinapoléonienne jusqu'à Larra - ce genre littéraire qui était la satire et que l'on suppose mineur, s'est converti en protagoniste de premier plan de la vie littéraire» (p. 20). L'étude d'A. Pérez est structurée en dix chapitres qui suivent, en même temps, un ordre chronologique. Ainsi, l'auteur part des années d'étude de médecine à Salamanque, de ses traductions et publications d'oeuvres de thème médique, et de son rapport avec les cinq « idéologues » français - signalant l'influence de ceux-ci sur une certaine dimension matérialiste du romantisme- en nous rappelant qu'en 1801, il avait été logeur des 
troupes de l'armée républicaine française - raison supplémentaire de son rapport direct avec la France. De retour à Salamanque, Gallardo a été en contact avec des personnages remarquables des Lumières espagnoles (tels Tavira ou Meléndez). Après un bref chapitre sur les premiers écrits satiriques, l'auteur souligne l'importance du bouleversement de la guerre antinapoléonienne (moment où Gallardo écrit son Apologia de los palos, peu après avoir été nommé bibliothécaire des Cortes de Cadix, en 1811). On arrive ainsi au chapitre central de l'étude d'A. Pérez, celui dédié au Diccionario criticoburlesco. Il s'agit d'une étude définitive sur ce texte paradigmatique de la polémique entre réaction et révolution dans le contexte des Cortes de Cadix - réponse satirique et burlesque à la publication du Diccionario razonado manual (1811), étudié aussi par A. Pérez dans le même chapitre. Ce dernier texte avait été l'expression de la volonté de disqualification politique du libéralisme, à travers une idée très grave dans le contexte de guerre contre la France, celle de l'identification des Lumières, du libéralisme et de la philosophie avec la France, et des idées réactionnaires avec celles authentiquement espagnoles et anti-françaises. À travers une minutieuse recherche, A. Pérez anéantit les mythes sur l'identité de l'auteur du Diccionario crifico-burlesco, ainsi que sur le supposé caractère conspiratif de son inspiration; nous montre le caractère politique - non doctrinal - des argumentations de Gallardo; souligne l'intérêt du Diccionario comme témoin d'une volonté d'affirmation laïque dans la pensée politique du premier libéralisme; et remarque une certaine ambiguïté qui est en même temps caractéristique de la révolution libérale espagnole (d'un côté la défense des principes révolutionnaires - les Cortes, la souveraineté du peuple, la Constitution, la liberté, l'égalité -, et de l'autre le besoin de s'éloigner de la Révolution française - mais sans une condamnation radicale du jacobinisme et des valeurs révolutionnaires...). À travers Gallardo, A. Pérez remarque aussi un trait spécifique, et très souvent interprété de manière simpliste, de la révolution espagnole : la façon à travers laquelle les libéraux espagnols ont cherché intentionnellement - à cause des circonstances et de la pression contre-révolutionnaire - les références religieuses et nationales, tout en laissant en arrière-plan les argumentations directement « scientifiques » ou objectives en défense de leurs idées politiques.

4 L'étude d'A. Pérez continue dans les chapitres suivants, où il étudie minutieusement l'activité de Gallardo comme journaliste, son exil à Londres après la restauration absolutiste de 1814, le retour et la présence discrète pendant le Trienio (1820-1823), et sa dédicace à l'étude philologique et littéraire, ainsi que son permanent anticonformisme politique, jusqu'à sa mort en 1852.

On est ici face à une contribution décisive sur une oeuvre et une figure fondamentales pour la compréhension de la révolution libérale espagnole - particulièrement en ce qui concerne l'affrontement politique entre révolution et réaction, ainsi qu'au rôle joué dans ce processus par la littérature.

On doit regretter, seulement, que la bonne qualité formelle de ce volume ne se trouve en correspondance avec une évidente pauvreté "instrumentale » de l'édition: pas de répertoire bibliographique, d'index onomastique et pas davantage de chronologie biobibliographique de B.Gallardo, qui auraient multiplié infiniment l'utilité de ce magnifique travail d'Alejandro Pérez Vidal. 